\title{
Natural transverse vibrations of helical springs in sections covered with elastic coatings
}

\author{
K. MICHALCZYK* \\ AGH University of Science and Technology, Department of Machine Design and Technology, 30 Mickiewicza Ave., 30-059 Krakow, Poland
}

\begin{abstract}
It has been demonstrated in previous studies that local elastomer coatings covering the end coils of helical springs can efficiently reduce the amplitudes of circum-resonant vibrations in such springs. The analysis of the influence that elastic coatings have on the frequencies and modes of natural transverse vibrations of springs is presented in this paper. The concept of the equivalent beam of the Timoshenko type is utilized in calculations of the frequencies and modes of transverse vibrations. The model developed allows users to determine the frequencies and modes of symmetric as well as antisymmetric vibrations of axially loaded springs with elastic coatings of arbitrary length. A comparison of the results obtained using FEM analysis, in which the model represented the actual spring geometry, with the results obtained by means of the presented model indicates its high accuracy.
\end{abstract}

Key words: helical spring vibrations, natural frequencies, free transverse vibrations, axially loaded spring, spring with coatings.

\section{Introduction}

Helical springs, widely used in machine building [1-4], are often exposed to operations under the influence of dynamic forces of a wide range of frequencies. Work under circum-resonant conditions, due to the negligible damping properties of steel, can cause spring vibrations of high amplitudes. Dynamic stresses formed then constitute threats for the work safety of springs. In order to limit the amplitudes of vibrations, methods based on increasing the energy scattering of spring vibrations are applied. Such a scattering effect can be obtained by means of external friction between a spring wire and cooperating elements [5] or by applying an elastic washer - made of a highly damping material - under a spring [6]. The drawback of the first solution is that it wipes off an external layer of a spring wire, which can negatively influence its fatigue strength, since the state of the spring wire surface is essential for its fatigue strength [7]. Meanwhile, the second solution increases the spring flexibility to its elastic stability, which was analyzed in [8]. Another solution increasing the scattering of spring vibration energy is to cover the spring wire with coatings made of a highly damping material, presented e.g. [9]. This method's efficiency in relation to longitudinal vibrations was investigated in [10]. It was shown in [11] that, with the application of the same amount of damping materials, better damping efficiency of longitudinal vibrations can be obtained by applying local elastomeric coatings. The influence of these coatings on the frequencies and modes of longitudinal vibrations was investigated in the same paper.

The analysis of problems related to the dynamic characteristics of springs can be carried out on three model types: the

*e-mail:kmichal@agh.edu.pl

Manuscript submitted 2017-03-29, revised 2017-04-14, initially accepted for publication 2017-04-19, published in December 2017. model of a spatially curved rod, the model of an equivalent rod or beam, and the model in which a continuous system, such as a spring, is substituted by a discrete-periodic system consisting of concentrated masses joined by a mass-less stiffness, jointly representing one spring coil or its fragment.

Equations of motion are written for an elementary fragment of a spring wire in the first model. Such a formulation of the problem [12,13] allows arbitrary modes and frequencies of spring vibrations to be determined, however, solving it by analytical methods is very difficult and therefore such a solution is not suitable in practical applications. These difficulties mean that in several studies concerning the analysis of spring vibrations treated as a spatially curved rod, the authors use numerical methods. New finite elements, capable of modeling spring coils or their fragments were proposed in [14]. They are suitable for application in static problems as well as when looking for natural frequencies of helical springs. Mottershead provided, in the above-mentioned paper, experimental results later utilized by other authors. A similar approach to the problems of helical springs was presented in [15-17]. Pearson expanded - in [18] - equations provided by Wittrick [13], taking into account the influence of a static axial force. For determining natural vibrations, he applied the transfer matrix method. The same method was used in [19], concerning the analysis of parameters influencing natural frequencies of helical springs (utilizing the Cayley-Hamilton theorem), and in [20]. In this last paper, the authors compared their results with the results obtained on the basis of the Haringx model [21]. They pointed out that, in the case of helical springs with geometrical parameters, typical of springs used in machine buildings, the conformity between the results obtained by them and the results obtained using the Haringx model is high. In the numerical example quoted by them, for which the first 16 natural frequencies were determined, the maximum difference between their results and the ones from the Haringx model did not exceed $1.3 \%$. In the case 
of springs of large helix angles and a high slenderness ratio (not generally applied in practice due to their tendency to lose elastic stability), the authors proved that in order to achieve high conformity between natural frequencies obtained by them and the results from the standard Haringx model, it is enough to multiply the last ones by the cosine of the helix angle. In the numerical example quoted by them, in which they determined the first 10 natural frequencies of the spring - with the helix angle at $23^{\circ}$ - after multiplication of the natural frequencies obtained from the Haringx model by the cosine of this angle, the differences between the results obtained from both models did not exceed $1 \%$. The authors noticed that more accurate dependencies, describing equivalent bending and shear stiffness, and applied in relation to the Haringx concept, would be able to explain this effect. Such dependencies, taking into account, among others, the non-zero and variable (together with an axial load-) values of the helix angle, in relation to the elastic stability problem of helical springs, were derived in [22]. A new numerical method of determining natural frequencies of helical springs by means of the stiffness method was proposed in [23]. The dynamic stiffness method involving the Wittrick-Williams algorithm [24] was applied for the accurate solution of the natural frequencies problem, in [25]. The authors of that study treated the spring wire as the Euler-Bernoulli beam. The same method was applied in [26] for the determination of natural frequencies of a spring whose wire was treated as the Timoshenko beam, taking into account the effects related to wire shearing and rotational inertia of its cross-sections. The authors compared the results thus obtained with the results given by Pearson in [18] and with the FEM results. The pseudospectral method was applied in [27] for natural frequencies determination. The authors of [28] presented models allowing the natural frequencies of springs made of wires of non-circular cross-sections to be determined, taking into consideration the influence of the warping effect (related to twisting of the spring wire) on natural frequency values. They showed that this influence was larger in the case of non-cylindrical springs than in the case of cylindrical ones.

A model treating the spring as an equivalent rod was given by Timoshenko [29]. Using this model, Timoshenko analyzed the problem of elastic stability of helical springs, assuming that the helix angle was constant and equaled zero, regardless of the axial load. Haringx used this model in his study concerning the elastic stability of helical springs [30] as well as in later works concerning vibrations of helical springs [21]. The results presented in this last study, concerning springs of various slenderness ratios and various relative deflections, link the zeroing of the first natural transverse frequency with the moment of losing static elastic stability (cf. [21], p. 80). This model was used and developed by Wittrick [13], who took into account couplings between longitudinal and torsional spring vibrations. He directed his attention to different wave propagation velocities along the helix line related to the wire twisting and bending. The authors of [31] expanded the studies of Haringx to higher frequencies of transverse vibrations of helix springs, relating their values to the value of the first longitudinal natural frequency and verifying the results obtained experimentally. They mentioned in this study that for certain values of relative spring shortening caused by its axial loading, equalizing of various mode frequencies of natural vibrations can occur. The authors of [32] analyzed the compatibility of results for frequencies of natural longitudinal and torsional vibrations obtained from the Haringx model, the Wittrick model and the equivalent discrete model with experimental results. They pointed out that, in relation to longitudinal forms of vibrations, a divergence between the results obtained from the Wittrick and the Haringx models becomes significant only for the mode of vibrations in which the wave length is smaller than approximately four coils. They also proved that for longitudinal and torsional vibrations the equivalent discrete-periodic model provides highly accurate results even for the form of the wave length that equals two coils. This model is used in engineering practice in the automotive industry for modeling dynamics of valve systems in internal combustion engines, and presented e.g. [33]. The Haringx model was also used in study [34] to determine the natural frequencies of transverse vibrations of a simply supported spring. It was pointed out, in [35], that the above-mentioned equivalent rod concept, proposed in [22] for the analysis of elastic stability of helical springs, can also be applied in the analysis of natural transverse vibrations of helical springs. Its application, after modification resulting from different boundary conditions, provides results nearer to FEM results and the results obtained by other authors $[18,26]$ (treating the spring as a spatially curved rod) than the standard concept of Timoshenko. A method that allows users to determine - before starting computations - the form of the solution for the wave equation of motion, depending on the spring slenderness ratio and its relative static deflection, was presented in this paper. This form, as it is known, depends on whether the investigated natural frequency of transverse vibrations is smaller or larger than the cut-off frequency. This problem was analyzed in relation to stocky beams in $[36,37]$.

The aim of this paper is to develop a model enabling users to determine the influence of the parameters of elastic coatings of arbitrary length, made of materials such as rubber or macromolecular polymers, on the frequencies and modes of natural transverse vibrations in springs loaded by a static axial force.

\section{Motion equations}

As it was shown in study [10] with reference to longitudinal vibrations of helical spring with damping coating covering the spring wire on its whole length, the influence of damping, which can be possible to obtain in practice by means of using coating materials such as rubber or typical macromolecular polymers, on natural frequencies is negligible. On the other hand, internal material damping - modeled on equivalent internal linear damping - has no influence on the shapes of natural modes of transversal vibrations. Thus, analysis of natural transversal vibrations of a helical spring with coatings made of elastic materials exhibiting damping properties can be conducted using a model of undamped vibrations without making significant mistakes. Further considerations are conducted under this assumption. 
The first form of transverse vibrations of a helical spring with local coatings loaded by force $P$ is presented in Fig. 1 . A fragment of the equivalent beam, of the Timoshenko type, has been superimposed onto the spring image.

For each of the three segments of the equivalent beam of lengths $L_{1}, L_{2}, L_{3}$, the following equations of motion can be written [21]:

$$
\begin{array}{rr}
\frac{\partial N_{i}}{\partial \psi_{i}}+Q_{i}=0 & i=1,2,3 \\
m_{i} \frac{\partial^{2} y_{i}}{\partial t^{2}}=N_{i} \frac{\partial \psi_{i}}{\partial x}-\frac{\partial Q_{i}}{\partial x} & i=1,2,3 \\
m_{i} r_{g}^{2} \frac{\partial^{2} \psi_{i}}{\partial t^{2}}=N_{i} \varphi_{i}+Q_{i}+\frac{\partial M_{i}}{\partial x} & i=1,2,3 .
\end{array}
$$

The $\psi_{i}$ angles are angles caused only by bending, while the $\varphi_{i}$ angles are angles resulting only from shearing relevant segments of the equivalent beam, which are described by the following equations:

$$
\begin{aligned}
& \psi_{i}+\varphi_{i}=-\frac{\partial y_{i}}{\partial x} \quad \psi_{i}=-\frac{\partial y_{\psi i}}{\partial x} \quad \varphi_{i}=-\frac{\partial y_{\varphi i}}{\partial x} \\
& \frac{\partial \psi_{i}}{\partial x}=\frac{M_{i}}{\alpha_{i}} \quad \varphi_{i}=\frac{Q_{i}}{\beta_{i}} \quad i=1,2,3 .
\end{aligned}
$$

Coefficients $\alpha_{i}$ and $\beta_{i}$ constitute the actual equivalent stiffness of the selected beam segment for bending and shearing, respectively. These coefficients can be expressed by means of the equations proposed in [22] and applied and modified in paper [35]. These dependencies, with the assumption that the number of spring coils is either total or half, can be presented in the following form:

$$
\begin{aligned}
& \alpha_{1}=\frac{2\left(E_{s} J_{s}+E_{c} J_{c}\right) \sin \delta_{1}}{2+\left(\frac{\left(E_{s} J_{s}+E_{c} J_{c}\right)\left(1+v_{s}\right)\left(1+v_{c}\right)}{E_{s} J_{s}\left(1+v_{c}\right)+E_{c} J_{c}\left(1+v_{s}\right)}-1\right) \cos ^{2} \delta_{1}} \\
& \alpha_{2}=\frac{2 E_{s} J_{s} \sin \delta_{2}}{2+v_{s} \cos ^{2} \delta_{2}} \\
& \beta_{1}=\frac{2 \sin \delta_{1} \cdot\left(E_{s} J_{s}+E_{c} J_{c}\right) / R^{2}}{1+\left(\frac{\left(E_{s} J_{s}+E_{c} J_{c}\right)\left(1+v_{s}\right)\left(1+v_{c}\right)}{E_{s} J_{s}\left(1+v_{c}\right)+E_{c} J_{c}\left(1+v_{s}\right)}-1\right) \sin ^{2} \delta_{1}} \\
& \beta_{2}=\frac{2 E_{s} J_{s} \sin _{2}}{R^{2}\left(1+v_{s} \sin ^{2} \delta_{2}\right)}
\end{aligned}
$$

where: $E_{s, c}$ - Young's modulus of steel (index "s") and of coating material (index "c"), $J_{s, c}$ - moment of inertia of the cross-section of the spring wire (index " $s$ ") and coating

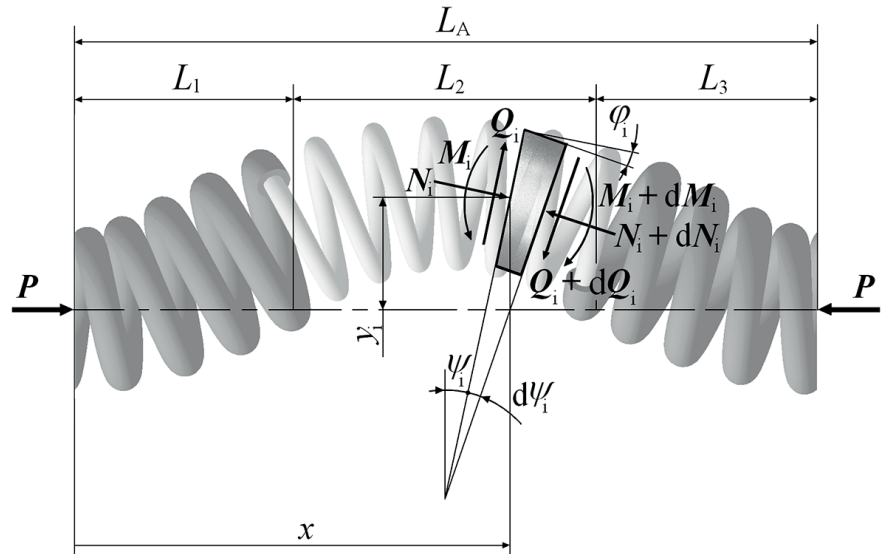

Fig. 1. Model of an equivalent rod to be used for the analysis of transverse vibrations of a helical spring with local damping coatings

(index “c"), respectively, $v_{s, c}$ - Poisson's ratio (indexes as in Young's modulus), $R$ - nominal spring radius (it was assumed that changes in its value during compression are negligible), $\delta_{1}$, $\delta_{2}-$ actual helix angles of the spring segment with coating and the spring segment without coating, respectively. These angles, due to the static force $P$ 's operation, can differ on account of different stiffness of each segment and will be expressed as:

$$
\delta_{i}=\arcsin \left(\left(1-P / \gamma_{0 i}\right) \cdot \sin \delta_{0}\right) \quad i=1,2
$$

where: $\delta_{0}$ - helix angle of an unloaded spring $(P=0)$, while compression stiffness $\gamma_{0 i}(i=1,2)$ is expressed as the following dependencies:

$$
\begin{aligned}
\gamma_{01} & =\frac{\sin \delta_{0} \cdot\left(E_{s} J_{s}+E_{c} J_{c}\right) / R^{2}}{1+\left(\frac{\left(E_{s} J_{s}+E_{c} J_{c}\right)\left(1+v_{s}\right)\left(1+v_{c}\right)}{E_{s} J_{s}\left(1+v_{c}\right)+E_{c} J_{c}\left(1+v_{s}\right)}-1\right) \cos ^{2} \delta_{0}} \\
\gamma_{02} & =\frac{E_{s} J_{s} \sin \delta_{0}}{R^{2}\left(1+v_{s} \cos ^{2} \delta_{0}\right)}
\end{aligned}
$$

Values $y_{\psi i}$ and $y_{\varphi i}$ occurring in (2) are displacements resulting only from bending and only from shearing, respectively. The actual linear density of the selected spring segment is denoted by $m_{i}(i=1,2,3)$. Considering inertia properties of the equivalent beam, it is assumed that the equivalent beam has the shape of a thin-walled cylinder with mean diameter equal to nominal spring diameter, as it satisfactorily approximates spring mass distribution in a plane normal to its axis. The mass radius of gyration of the equivalent beam cross-section $r_{\mathrm{g}}$ is assumed as constant at its whole length and independent of its axial loading by force $P$

$$
r_{g}=R \sqrt{2} / 2
$$

Radius $R$ in (7) equals half of the nominal spring diameter. It was assumed in the calculations that its value was constant and independent of axial force $P$. The values of normal force $N_{i}$ 
for individual spring segments are: $N_{i}=P \cos \psi_{i}$. After transformations made in the same way as in [21] and later in [31] and [35], we can obtain three equations $(i=1,2,3)$ of transverse vibrations of spring segments:

$$
\begin{aligned}
& \frac{\partial^{4} \psi_{i}}{\partial x^{4}}-\left(\frac{m_{i}}{\beta_{i}}+\frac{m_{i} r_{g}^{2}}{\alpha_{i}}\right) \frac{\partial^{4} \psi_{i}}{\partial x^{2} \partial t^{2}}+\frac{m_{i}^{2} r_{g}^{2}}{\alpha_{i} \beta_{i}} \frac{\partial^{4} \psi_{i}}{\partial t^{4}}+ \\
& +\frac{P}{\alpha_{i}}\left(1+\frac{P}{\beta_{i}}\right) \frac{\partial^{2} \psi_{i}}{\partial x^{2}}+\frac{m_{i}}{\alpha_{i}}\left(1+\frac{P}{\beta_{i}}\right) \frac{\partial^{2} \psi_{i}}{\partial t^{2}}=0 .
\end{aligned}
$$

Calculations for three spring segments would require the solving of twelve equations. However, assuming the same parameters of coatings on both spring ends - which is fully justified from the point of view of maximum damping efficiency [11] - the same results can be obtained when considering only half of the spring (for $x$ belonging to the interval of 0 to $L_{A} / 2$ ) and imposing proper boundary conditions, which leads to the necessity of solving only eight equations. Since transverse vibrations of clamped-clamped springs can have either a symmetrical form (forms of odd numbers: $1,3,5, \ldots$ ) or an anti-symmetrical form (forms of even numbers: $2,4,6, \ldots$ ), it should be remembered that there is a change - depending on the number of the desired frequency of vibrations - in boundary conditions at the right end of the spring model considered (for $x=L_{A} / 2$ ). These conditions will be discussed later in this paper. Thus, further considerations are limited to the analysis of the left half of the spring presented in Fig. $1(i=1,2)$.

Using the Bernoulli-Fourier method, functions $\psi_{i}(x, t), \varphi_{i}(x$, $t)$ and $y_{i}(x, t)$ can be written in the following form:

$$
\begin{aligned}
& \psi_{i}(x, t)=\Psi_{i}(x) \sin \omega t \quad \varphi_{i}(x, t)=\Phi_{i}(x) \sin \omega t \\
& y_{i}(x, t)=Y_{i}(x) \sin \omega t \quad i=1,2
\end{aligned}
$$

where $\Psi_{i}(x)$ are amplitudes of angles resulting only from bending, $\Phi_{i}(x)$ are amplitudes of angles resulting only from shearing, $Y_{i}(x)$ are total displacement amplitudes and $\sin \omega t$ is a desired time function.

By substituting the relevant derivatives of (9) to (8) and rearranging, we obtain the following equation:

$$
\Psi_{i} \mathrm{IV}+b_{i} \Psi_{i} \mathrm{II}-c_{i} \Psi_{i}=0
$$

where [35]:

$$
\begin{aligned}
b_{i} & =\left(\frac{m_{i}}{\beta_{i}}+\frac{m_{i} r_{g}^{2}}{\alpha_{i}}\right) \omega^{2}+\frac{P}{\alpha_{i}}\left(1+\frac{P}{\beta_{i}}\right) \\
c_{i} & =\frac{m_{i}}{\alpha_{i}}\left(1+\frac{P}{\beta_{i}}\right) \omega^{2}-\frac{m_{i}^{2} r_{g}^{2}}{\alpha_{i} \beta_{i}} \omega^{4}
\end{aligned}
$$

The form of the solution of (10) depends on whether the frequency value $\omega$ is higher or lower than the values of cut-off frequencies $\omega_{i b}$. Investigations performed in [35] have shown that even for relatively stocky springs, the first natural frequencies of transverse vibrations are usually lower than the cut-off frequency. Therefore this case, i.e. the one most often met in practice, will be considered in the paper.

Thus, we are looking for solutions of (10) in the following form:

$$
\begin{aligned}
\Psi_{i}(x) & =C_{1 i} \cosh \left(k_{1 i} x\right)+C_{2 i} \sinh \left(k_{1 i} x\right)+\quad i=1,2 \\
& +C_{3 i} \cos \left(k_{2 i} x\right)+C_{4 i} \sin \left(k_{2 i} x\right)
\end{aligned}
$$

where:

$$
\begin{aligned}
& k_{1 i}=\sqrt{\frac{1}{2}\left(-b_{i}+\sqrt{b_{i}^{2}+4 c_{i}}\right)} \\
& k_{2 i}=\sqrt{\frac{1}{2}\left(b_{i}+\sqrt{b_{i}^{2}+4 c_{i}}\right)} .
\end{aligned}
$$

Using (1), (2), (9) and (12) after proper transformations, we obtain total displacement amplitude equations for the first and second element of the spring:

$$
\begin{aligned}
Y_{i}(x) & =A_{1 i}\left(C_{1 i} \sinh k_{1 i} x+C_{2 i} \cosh k_{1 i} x\right)+ \\
& +A_{2 i}\left(C_{3 i} \sin k_{2 i} x-C_{4 i} \cos k_{2 i} x\right)
\end{aligned} \quad i=1,2
$$

where:

$$
\begin{aligned}
& A_{1 i}=\frac{-P k_{1 i}}{m_{i} \omega^{2}}-\frac{r_{g}^{2} \beta_{i} k_{1 i}}{\beta_{i}+P}-\frac{\alpha_{i} \beta_{i} k_{1 i}^{3}}{m_{i} \omega^{2}\left(\beta_{i}+P\right)} \\
& A_{2 i}=\frac{P k_{2 i}}{m_{i} \omega^{2}}-\frac{r_{g}^{2} \beta_{i} k_{2 i}}{\beta_{i}+P}-\frac{\alpha_{i} \beta_{i} k_{2 i}^{3}}{m_{i} \omega^{2}\left(\beta_{i}+P\right)} .
\end{aligned}
$$

In a similar way, substituting the relevant derivatives of $(9)_{1}$ with (1) and (2) and using (9) 2 and (12), we obtain - after rearrangements - equations of amplitudes of the angle of deflection resulting from shearing only:

$$
\begin{aligned}
\Phi_{i}(x) & =A_{3 i}\left(C_{1 i} \cosh k_{1 i} x+C_{2 i} \sinh k_{1 i} x\right)+\quad i=1,2 \\
& +A_{4 i}\left(C_{3 i} \cos k_{2 i} x+C_{4 i} \sin k_{2 i} x\right)
\end{aligned}
$$

where:

$$
A_{3 i}=\frac{-m_{i} r_{g}^{2} \omega^{2}-\alpha_{i} k_{1 i}^{2}}{\beta_{i}+P} \quad A_{4 i}=\frac{-m_{i} r_{g}^{2} \omega^{2}+\alpha_{i} k_{2 i}^{2}}{\beta_{i}+P}
$$

Equations (12), (14) and (16) allow frequencies and modes of transverse vibrations of the spring with local coatings to be determined, for the given boundary conditions.

Utilizing $(9)_{1,2},(12)$ and (16) in $(2)_{2,3}$, we obtain - after rearrangements and integrations - equations describing the displacement amplitudes resulting only from bending $\left(Y_{\psi i}\right)$ and the 
displacement amplitudes resulting only from shearing $\left(Y_{\Phi i}\right)$ the equivalent beam:

$$
\begin{aligned}
& Y_{\Psi i}(x)=\left(C_{4 i} \cos k_{2 i} x-C_{3 i} \sin k_{2 i} x\right) / k_{2 i}- \\
& -\left(C_{1 i} \sinh k_{1 i} x+C_{2 i} \cosh k_{1 i} x\right) / k_{1 i}+C_{\Psi i} \\
& i=1,2
\end{aligned}
$$

$Y_{\Phi i}(x)=A_{4 i}\left(C_{4 i} \cos k_{2 i} x-C_{3 i} \sin k_{2 i} x\right) / k_{2 i}-$

$-A_{3 i}\left(C_{1 i} \sinh k_{1 i} x+C_{2 i} \cosh k_{1 i} x\right) / k_{1 i}+C_{\Phi i} i=1,2$.

Equations (18) and (19) allow the influence of shearing the equivalent beam (modeling the spring) on the total spring displacement amplitude to be determined. Integration constants $C_{\Psi i}$ and $C_{\Phi i}$ will be determined below, separately for symmetrical and anti-symmetrical forms of vibrations.

\section{Symmetrical forms of vibrations}

Utilizing the conditions: $Y_{\Psi_{1}}(0)=Y_{\Phi 1}(0)=0$ as well as $Y_{\Psi 1}\left(L_{1}\right)=Y_{\Psi 2}\left(L_{1}\right)$ and $Y_{\Psi 2}\left(L_{1}\right) Y_{\Phi 1}\left(L_{1}\right)=Y_{\Phi 2}\left(L_{1}\right)$, we find integration constants $C_{\Psi_{i}}$ and $C_{\Phi i}$, occurring in (18) and (19):

$$
\begin{aligned}
C_{\Psi 1} & =\frac{C_{21}}{k_{11}}-\frac{C_{41}}{k_{21}} \quad C_{\Phi 1}=\frac{A_{31} C_{21}}{k_{11}}-\frac{A_{41} C_{41}}{k_{21}} \\
C_{\Psi 2} & =Y_{\Psi 1}\left(L_{1}\right)-\frac{C_{42} \cos k_{22} L_{1}-C_{32} \sin k_{22} L_{1}}{k_{22}}+ \\
& +\frac{C_{12} \sinh k_{12} L_{1}+C_{22} \cosh k_{12} L_{1}}{k_{12}} \\
C_{\Phi 2} & =Y_{\Phi 1}\left(L_{1}\right)-\frac{A_{42}}{k_{22}}\left(C_{42} \cos k_{22} L_{1}-C_{32} \sin k_{22} L_{1}\right)+ \\
& +\frac{A_{32}}{k_{12}}\left(C_{12} \sinh k_{12} L_{1}+C_{22} \cosh k_{12} L_{1}\right) .
\end{aligned}
$$

For the considered model of the clamped-clamped spring half and the desired odd natural frequencies $(1,3,5, \ldots)$, the boundary conditions are as follows:
1. $y_{1}(0, t)=0$
2. $\psi_{1}(0, t)=0$
3. $\psi_{2}\left(L_{h}, t\right)=0$
4. $\varphi_{2}\left(L_{h}, t\right)=0$

where $L_{h}=L_{A} / 2$. Compatibility conditions, where the left coating ends with $\left(x=L_{1}\right)$ in Fig. 1, take the following forms:

$$
\begin{aligned}
& \text { 5. } \psi_{1}\left(L_{1}, t\right)=\psi_{2}\left(L_{1}, t\right) \quad \text { 6. } y_{1}\left(L_{1}, t\right)=y_{2}\left(L_{1}, t\right) \\
& \text { 7. }\left.\alpha_{1} \frac{\partial \psi_{1}(x, t)}{\partial x}\right|_{x=L_{1}}=\left.\alpha_{2} \frac{\partial \psi_{2}(x, t)}{\partial x}\right|_{x=L_{1}}
\end{aligned}
$$

8. $\beta_{1} \varphi_{1}\left(L_{1}, t\right)=\beta_{2} \varphi_{2}\left(L_{1}, t\right)$.
Using conditions: $\mathbf{1}, \mathbf{2}, \mathbf{3}, \mathbf{4}, \mathbf{5}, \mathbf{6}, \mathbf{7}$, the constants occurring in (12) were calculated. Constant $C_{11}$ was assumed as the constant of a known value. On account of the complex form of the coefficients being calculated, the following notations were introduced for simplification purposes:

$$
\begin{aligned}
B_{1}= & \frac{\cosh k_{11} L_{1}-\cos k_{21} L_{1}}{\cos k_{22} L_{1}-\frac{\sin k_{22} L_{1}}{\tan k_{22} L_{h}}} \\
B_{2}= & \frac{\sinh k_{11} L_{1}+\frac{A_{11}}{A_{21}} \sin k_{21} L_{1}}{\sin k_{22} L_{1}} \\
B_{3}= & \frac{\cos k_{22} L_{1}-\frac{\cosh k_{12} L_{1}-\frac{\sinh k_{12} L_{1}}{\tanh k_{12} L_{h}}}{\sin k_{22} L_{1}}}{\tan k_{22} L_{h}} \\
B_{4}= & \sinh k_{12} L_{1}-\frac{\cosh k_{12} L_{1}}{\tanh k_{12} L_{h}} \\
B_{5}= & \sin k_{22} L_{1}+\frac{\cos k_{22} L_{1}}{\tan k_{22} L_{h}} \\
B_{6}= & A_{11} \sinh k_{11} L_{1}-A_{21} \sin k_{21} L_{1}-B_{1} B_{5} A_{22} \\
B_{7}= & A_{11}\left(\cosh k_{11} L_{1}-\cos k_{21} L_{1}\right)-B_{2} B_{5} A_{22} \\
B_{8}= & B_{4} A_{12}-B_{3} B_{5} A_{22} \cdot
\end{aligned}
$$

After introducing the above notations, the constants occurring in (12) are as follows:

$C_{11}=$ idem

$C_{21}=\frac{\left(\begin{array}{l}\frac{\alpha_{1}}{\alpha_{2}}\left(k_{11} \cdot \sinh k_{11} L_{1}+k_{21} \cdot \sin k_{21} L_{1}\right)- \\ \left.-B_{4} B_{6} \frac{k_{12}}{B_{8}}+\left(\frac{B_{1}}{B_{3}}-\frac{B_{6}}{B_{8}}\right) B_{3} B_{5} k_{22}\right)\end{array}\right.}{\left(\begin{array}{l}\frac{-\alpha_{1}}{\alpha_{2}}\left(k_{11} \cdot \cosh k_{11} L_{1}+\frac{A_{11}}{A_{21}} k_{21} \cdot \cos k_{21} L_{1}\right) \\ +B_{4} B_{7} \frac{k_{12}}{B_{8}}-\left(\frac{B_{2}}{B_{3}}-\frac{B_{7}}{B_{8}}\right) B_{3} B_{5} k_{22}\end{array}\right)} C_{11}$

$C_{31}=-C_{11} \quad C_{41}=\frac{A_{11}}{A_{21}} C_{21}$

$C_{12}=\frac{B_{6}}{B_{8}} C_{11}+\frac{B_{7}}{B_{8}} C_{21} \quad C_{22}=\frac{-C_{12}}{\tanh k_{12} L_{h}}$

$C_{32}=B_{1} C_{11}+B_{2} C_{21}-B_{3} C_{12} \quad C_{42}=\frac{-C_{32}}{\tanh k_{22} L_{h}}$. 
Using the last condition from (22), the frequency equation for the spring model under consideration can be written in the following form:

$$
\begin{array}{r}
0=\beta_{1}\left(\begin{array}{l}
A_{31}\left(C_{11} \cosh k_{11} L_{1}+C_{21} \sinh k_{11} L_{1}\right)+ \\
+A_{41}\left(C_{31} \cos k_{21} L_{1}+C_{41} \sin k_{21} L_{1}\right)
\end{array}\right)- \\
-\beta_{2}\left(\begin{array}{l}
A_{32}\left(C_{12} \cosh k_{12} L_{1}+C_{22} \sinh k_{12} L_{1}\right)+ \\
+A_{42}\left(C_{32} \cos k_{22} L_{1}+C_{42} \sin k_{22} L_{1}\right)
\end{array}\right) .
\end{array}
$$

\section{Anti-symmetrical forms of vibrations}

Equations (18) and (19) can be rewritten in the same form in the case of anti-symmetrical vibrations, however coefficients appearing in them will be different and for clarity they will be marked with superscript " $a$ ". On account of anti-symmetrical vibrations, the conditions: $Y_{\Psi_{2}}\left(L_{h}\right)=Y_{\Phi 2}\left(L_{h}\right)=0$ as well as the compatibility conditions (as in the case of symmetrical vibrations) $Y_{\Psi_{1}}\left(L_{1}\right)=Y_{\Psi_{2}}\left(L_{1}\right)$ and $Y_{\Phi 1}\left(L_{1}\right)=Y_{\Phi 2}\left(L_{1}\right)$ must be met. Utilizing the above conditions in (18) and (19), constants $C_{\Psi i}^{a}$ and $C_{\Phi i}^{a}$ are calculated:

$$
\begin{aligned}
C_{\Psi 1}^{a} & =Y_{\Psi 2}\left(L_{1}\right)-\frac{C_{41}^{a} \cos \left(k_{21} L_{1}\right)-C_{31}^{a} \sin \left(k_{21} L_{1}\right)}{k_{21}}+ \\
& +\frac{C_{11}^{a} \sinh \left(k_{11} L_{1}\right)+C_{21}^{a} \cosh \left(k_{11} L_{1}\right)}{k_{11}} \\
C_{\Phi 1}^{a}= & Y_{\Phi 2}\left(L_{1}\right)-\frac{A_{41}}{k_{21}}\left(C_{41}^{a} \cos k_{21} L_{1}-C_{31}^{a} \sin k_{21} L_{1}\right)+ \\
+ & \frac{A_{31}}{k_{11}}\left(C_{11}^{a} \sinh k_{11} L_{1}-C_{21}^{a} \cosh k_{11} L_{1}\right) \\
C_{\Psi 2}^{a}= & \frac{C_{12}^{a} \sinh \left(k_{12} L_{h}\right)+C_{22}^{a} \cosh \left(k_{12} L_{h}\right)}{k_{12}}- \\
& -\frac{C_{42}^{a} \cos \left(k_{22} L_{h}\right)-C_{32}^{a} \sin \left(k_{22} L_{h}\right)}{k_{22}} \\
C_{\Phi 2}^{a}= & \frac{A_{32}}{k_{12}}\left(C_{12}^{a} \sinh k_{12} L_{h}+C_{22}^{a} \cosh k_{12} L_{h}\right)- \\
+ & \frac{A_{42}}{k_{22}}\left(C_{42}^{a} \cos k_{22} L_{h}-C_{32}^{a} \sin k_{22} L_{h}\right) .
\end{aligned}
$$

For frequencies of even numbers $(2,4,6, \ldots)$ the procedure is analogous, in addition to which the third and fourth conditions from (21) should be replaced by the following conditions:

$$
y_{2}\left(L_{h}, t\right)=\left.0 \quad \alpha_{2} \frac{\partial \psi_{2}(x, t)}{\partial x}\right|_{x=L_{\mathrm{h}}}=0
$$

Once more to simplify the equation we introduce the following notations:

$$
\begin{aligned}
& B_{1}^{a}=\frac{\cosh k_{11} L_{1}-\cos k_{21} L_{1}}{\cos k_{22} L_{1}+\tan k_{22} L_{h} \cdot \sin k_{22} L_{1}} \\
& B_{2}^{a}=\frac{\sinh k_{11} L_{1}+\frac{A_{11}}{A_{21}} \sin k_{21} L_{1}}{\cos k_{22} L_{1}+\tan k_{22} L_{h} \cdot \sin k_{22} L_{1}} \\
& B_{3}^{a}=\frac{\cosh k_{12} L_{1}-\tanh k_{12} L_{h} \cdot \sinh k_{12} L_{1}}{\cos k_{22} L_{1}+\tan k_{22} L_{h} \cdot \sin k_{22} L_{1}} \\
& B_{4}^{a}=\sinh k_{12} L_{1}-\tanh k_{12} L_{h} \cdot \cosh k_{12} L_{1} \\
& B_{5}^{a}=\sin k_{22} L_{1}-\tan k_{22} L_{h} \cdot \cos k_{22} L_{1} \\
& B_{6}^{a}=A_{11} \sinh k_{11} L_{1}-A_{21} \sin k_{21} L_{1}-B_{1}^{a} A_{22} B_{5}^{a} \\
& B_{7}^{a}=A_{11}\left(\cosh k_{11} L_{1}-\cos k_{21} L_{1}\right)-B_{2}^{a} A_{22} B_{5}^{a} \\
& B_{8}^{a}=A_{12} B_{4}^{a}-B_{3}^{a} A_{22} B_{5}^{a} .
\end{aligned}
$$

Constants from (12) can be expressed in the form

$$
\begin{aligned}
& C_{11}^{a}=\text { idem } \\
& C_{21}^{a}=\frac{\left(\begin{array}{l}
\frac{\alpha_{1}}{\alpha_{2}}\left(k_{11} \cdot \sinh k_{11} L_{1}+k_{21} \cdot \sin k_{21} L_{1}\right)- \\
\left.-\frac{B_{6}^{a}}{B_{8}^{a}} k_{12} B_{4}^{a}+\left(B_{1}^{a}-\frac{B_{6}^{a}}{B_{8}^{a}} B_{3}^{a}\right) k_{22} B_{5}^{a}\right)
\end{array}\right.}{\left(\begin{array}{l}
\frac{-\alpha_{1}}{\alpha_{2}}\left(k_{11} \cdot \cosh k_{11} L_{1}+\frac{A_{11}}{A_{21}} k_{21} \cdot \cos k_{21} L_{1}\right)+ \\
+\frac{B_{7}^{a}}{B_{8}^{a}} k_{12} B_{4}^{a}-\left(B_{2}^{a}-\frac{B_{7}^{a}}{B_{8}^{a}} B_{3}^{a}\right) k_{22} B_{5}^{a}
\end{array}\right)} C_{11}^{a} \\
& C_{31}^{a}=-C_{11}^{a} \quad C_{41}^{a}=\frac{A_{11}}{A_{21}} C_{21}^{a} \\
& C_{12}^{a}=\frac{B_{6}^{a}}{B_{8}^{a}} C_{11}^{a}+\frac{B_{7}^{a}}{B_{8}^{a}} C_{21}^{a} \quad C_{22}^{a}=-C_{12}^{a} \tanh k_{12} L_{h} \\
& C_{32}^{a}=B_{1}^{a} C_{11}^{a}+B_{2}^{a} C_{21}^{a}-B_{3}^{a} C_{12}^{a} \quad C_{42}^{a}=C_{32}^{a} \tan k_{22} L_{h} .
\end{aligned}
$$

The frequency equation has a form analogous to (25) with the exception of constants $C_{j i}(j=1,2,3,4 ; i=1,2)$ having to be replaced with constants $C_{j i}^{a}$.

\section{Simulations, results and discussion}

In order to illustrate the model presented herein, a spring of the following parameters was used: nominal diameter $D=100 \mathrm{~mm}$, wire diameter $d_{s}=10 \mathrm{~mm}$, initial pitch of a helix $h_{0}=40 \mathrm{~mm}$, total height of the non-loaded spring $L_{A 0}=380 \mathrm{~mm}$, initial 
height of elastic coatings $L_{10}=L_{30}=100 \mathrm{~mm}$, external diameter of coatings $d_{c}=18 \mathrm{~mm}$. Typical material properties of spring steel were assumed for the spring wire: Young's modulus $E_{s}=209000 \mathrm{MPa}$, Poisson's ratio $v_{s}=0.28$, density $\rho_{s}=7800 \mathrm{~kg} / \mathrm{m}^{3}$.

Viscoelastic materials, such as rubber, are generally applied as damping materials in machine building. It was shown in [11] that coatings made of materials of typical rubber parameters have significantly lower efficiency when damping a spring's natural vibrations than coatings made of materials with parameters typical for high-molecular plastics, such as HDPE, PP or PTFE. This is related to a very low - in comparison to steel - modulus of transverse stiffness, even in the case of rubbers of hardness exceeding $90^{\circ} \mathrm{Sh}$. Alongside the progress in plastic production, polymers and composite polymers, which are characterized by high damping, strength and stiffness properties, are more and more often applied in vibration damping systems $[38,39,40]$. On account of the fact that the properties of individual plastics can significantly differ depending on their production technology [41], work temperature, frequency and amplitudes of deformations, it was decided to assume approximate properties of the coating material typical for materials such as HDPE or PP, which can be found in [42] and [43]: Young's modulus $E_{c}=1430 \mathrm{MPa}$, Poisson's ratio $v_{c}=0.43$, density $\rho_{c}=1100 \mathrm{~kg} / \mathrm{m}^{3}$.
The modes and frequencies of natural vibrations obtained using the model presented herein were compared with the numerical simulation results obtained in the ANSYS Mechanical APDL environment. Solid models of the spring and the coatings were prepared directly in ANSYS, by creating helical paths and sweeping proper sections along them. Contact surfaces of the spring wire model and the coatings models were bonded together before the meshing process. Discretization of the model of the spring with coatings was carried out using 20-node non-layered SOLID186 structural elements. Modal analyses were performed using the Block Lanczos mode-extraction method. In the case of an axially loaded spring, the effects of pre-stress and large deflections were included.

5.1. Spring not subjected to axial loads. The first four forms of transverse vibrations of the clamped-clamped spring not subjected to axial loads $(P=0 \mathrm{~N})$, obtained from the modal FEM analysis, are presented in Fig. 2a, b, d, e. The first two vibration forms obtained using the model presented herein are shown in Fig. 2c, f. Thicker red lines in Fig. 2c, f illustrate displacement amplitudes of spring segments covered by coatings, while thinner black lines illustrate displacement amplitudes of spring segments without coatings. Dotted lines present displacement amplitudes $Y_{\Phi i}$, resulting from shearing of the equivalent beam, dashed lines present displacement amplitudes $Y_{\psi i}$, resulting from (a)
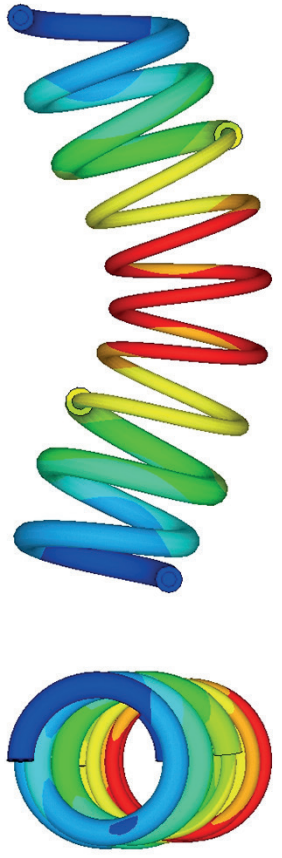

(b)

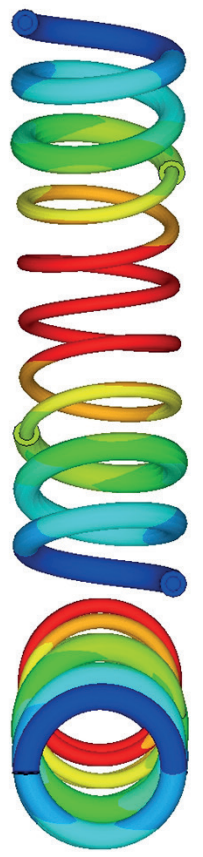

(c)

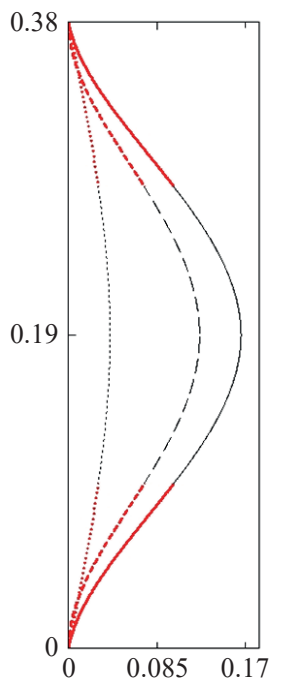

(d)

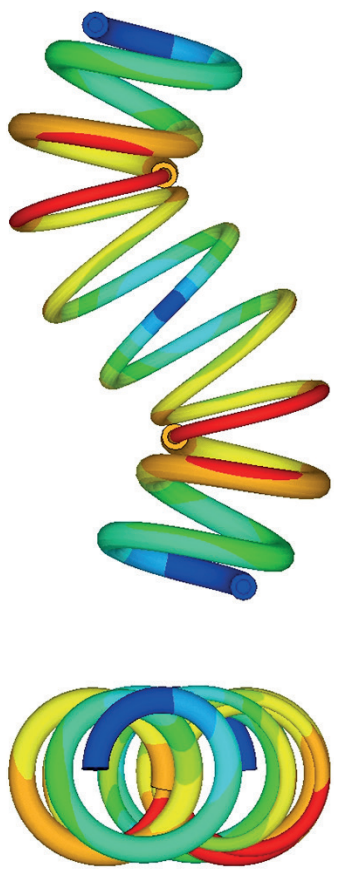

(e)

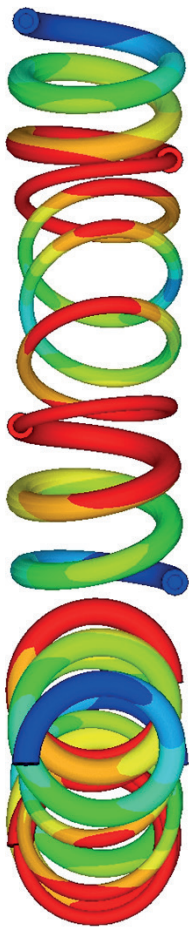

(f)

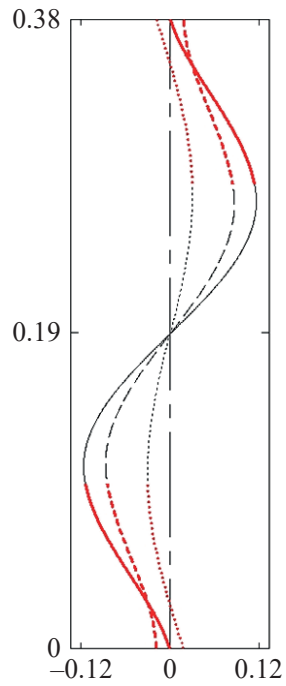

Fig. 2. First four forms of transverse vibrations of the clamped-clamped spring obtained from the FEM simulation (a, b, d, e) and first two forms of vibrations obtained on the basis of the model presented herein $(\mathrm{c}, \mathrm{f})$, of the spring that is non-loaded axially. Dotted lines in diagrams $\mathrm{c}$ and f represent displacement amplitudes resulting from shearing $Y_{\Phi}$, dashed lines represent displacement amplitudes resulting from bending $Y_{\psi i}$, while solid lines represent amplitudes of total displacements $Y_{i}$ of successive segments of the equivalent beam 
bending of the equivalent beam, and solid lines present the amplitudes $Y_{\mathrm{i}}$ of the total displacement.

The equivalent beam model, contrary to the FEM model representing the actual spring geometry as a spatially curved rod, exhibits the axial symmetry of stiffness and inertia parameters. Therefore, the first two frequencies of natural vibrations $f_{1}$ and $f_{2}$ of the spring modelled in the ANSYS environment, vibrating in two planes (Fig. 2a, b), correspond to one natural frequency $f_{\mathrm{I}}$ of the equivalent beam (Fig. 2c). The same is true for antisymmetric vibration forms. A comparison of frequencies obtained from the FEM simulation and frequencies obtained using the model presented herein is given in Table 1 .

Table 1

Comparison of natural frequencies (in $\mathrm{Hz}$ ) obtained from the FEM simulation with frequencies obtained from the model presented herein for a spring not subjected to an axial load

\begin{tabular}{|l|cccc|}
\cline { 2 - 5 } \multicolumn{1}{c|}{} & $f_{\mathbf{1}}$ & $\boldsymbol{f}_{\mathbf{2}}$ & $\boldsymbol{f}_{\mathbf{3}}$ & $\boldsymbol{f}_{\mathbf{4}}$ \\
\hline FEM model (Fig. 2a, b, d, e) & 30.981 & 31.074 & 66.882 & 67.565 \\
\hline $\begin{array}{l}\text { Model presented in this paper } \\
\text { (Fig. 2c, f) }\end{array}$ & $f_{\mathrm{I}}=31.126$ & $f_{\mathrm{II}}=68.232$ \\
\hline$\left(\left(f_{1,2,3,4}-f_{\mathrm{I}, \mathrm{II}}\right) / f_{1,2,3,4}\right) \cdot 100 \%$ & $-0.47 \%$ & $-0.17 \%$ & $-2.02 \%$ & $-0.99 \%$ \\
\hline
\end{tabular}

5.2. Spring loaded by static axial force. To analyze the modes and frequencies of natural vibrations of springs loaded by static axial force $P$, a pre-stressed modal analysis of a large-deflection solution was performed. A comparison of forms obtained from the simulation and from the model presented in the study for the spring loaded by static axial force $P=1046 \mathrm{~N}$, causing the spring shortening by $1 / 4$ of its initial length, is presented in Fig. 3.

A comparison of frequencies obtained from the FEM simulation with the frequencies obtained using the model presented herein for a spring compressed statically - by force $P=1046 \mathrm{~N}$ - to the length: $L_{A}=0.75 \cdot L_{A 0}$, is shown in Table 2 .

Table 2

Comparison of natural frequencies (in $\mathrm{Hz}$ ) obtained from the FEM simulation with frequencies obtained using the model presented herein for the axially loaded spring

\begin{tabular}{|l|cccc|}
\cline { 2 - 5 } \multicolumn{1}{c|}{} & $\boldsymbol{f}_{\mathbf{1}}$ & $\boldsymbol{f}_{\mathbf{2}}$ & $\boldsymbol{f}_{\mathbf{3}}$ & $\boldsymbol{f}_{\mathbf{4}}$ \\
\hline FEM model (Fig. 3a, b, d, e) & 30.672 & 30.765 & 67.325 & 68.283 \\
\hline $\begin{array}{l}\text { Model presented in this paper } \\
\text { (Fig. 3c, f) }\end{array}$ & $f_{\mathrm{I}}=30.885$ & $f_{\mathrm{II}}=69.246$ \\
\hline$\left(\left(f_{1,2,3,4}-f_{\mathrm{I}, \mathrm{II}}\right) / f_{1,2,3,4}\right) \cdot 100 \%$ & $-0.69 \%$ & $-0.39 \%$ & $-2.86 \%$ & $-1.41 \%$ \\
\hline
\end{tabular}

The data shown in Tables 1 and 2 indicate that the compatibility of results obtained using the model presented herein and numerical simulation is high, for the spring that is axially loaded as well as for the spring not subjected to axial loads.

\subsection{Influence of geometrical parameters of coatings on} natural frequencies. The diagram showing the dependence of the first natural frequency on relative height $L_{1} / L_{h}$ and relative thickness $\left(d_{c}-d_{s}\right) / d_{s}$ of elastic coatings, at the material parameters given above, is shown in Fig. 4, while the analogous diagram for the second natural frequency is shown in Fig. 5. Both diagrams are obtained using the analytical model presented in (a)
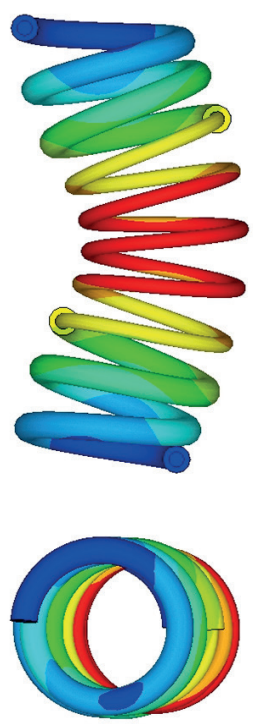

(b)

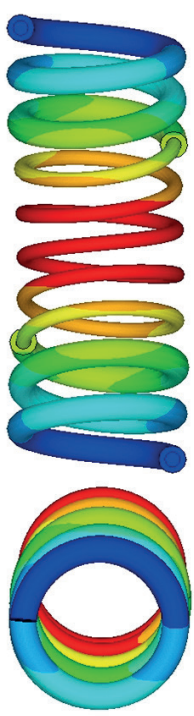

(c)

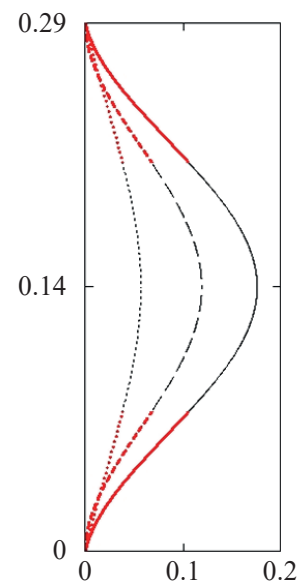

(d)

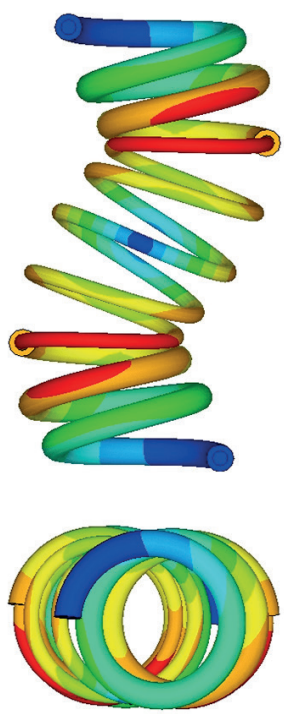

(e)

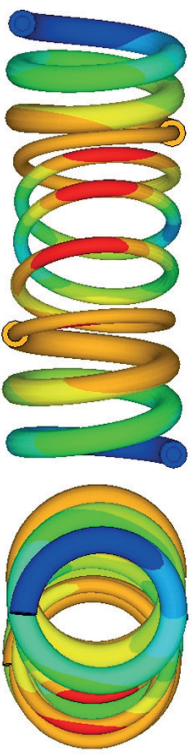

(f)

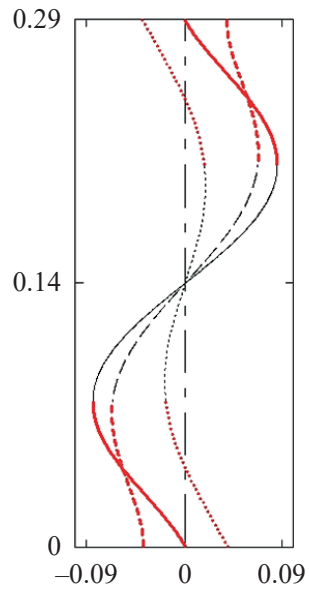

Fig. 3. First four forms of transverse vibrations of the clamped-clamped spring obtained from the FEM simulation (a, b, d, e) and first two forms obtained using the model presented herein (c, f), for the axially loaded spring $\left(P=1046 \mathrm{~N}, L_{A}=0.75 \cdot L_{A 0}=285 \mathrm{~mm}\right)$ 


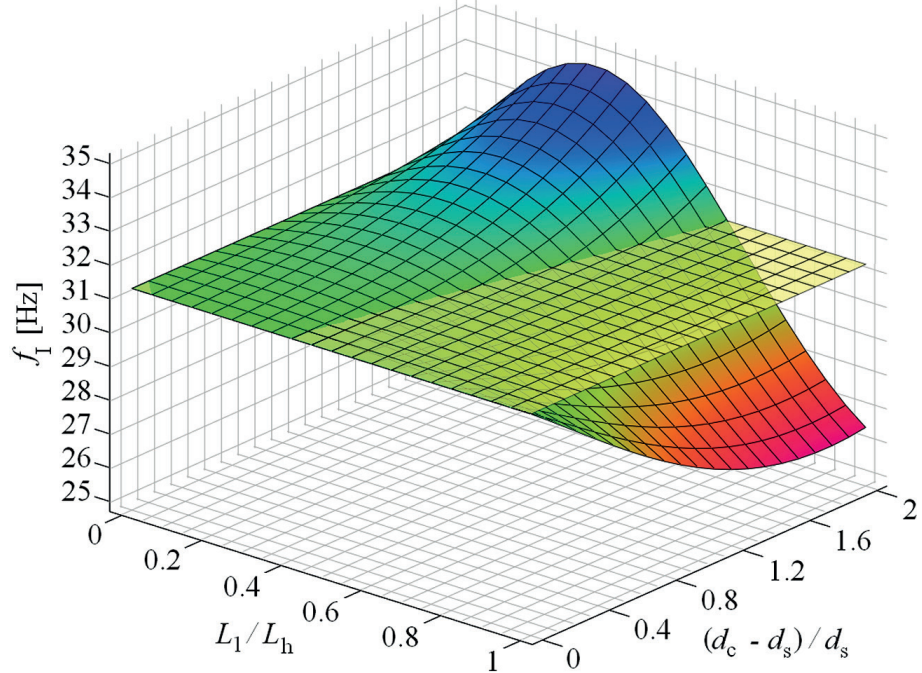

Fig. 4. Dependence of the first transverse natural frequency $f_{1}$ on the relative height of local coatings $\left(d_{c}-d_{s}\right) / d_{s}$ and on the relative thickness of local coatings $L_{1} / L_{h}$, at the determined material parameters of these coatings

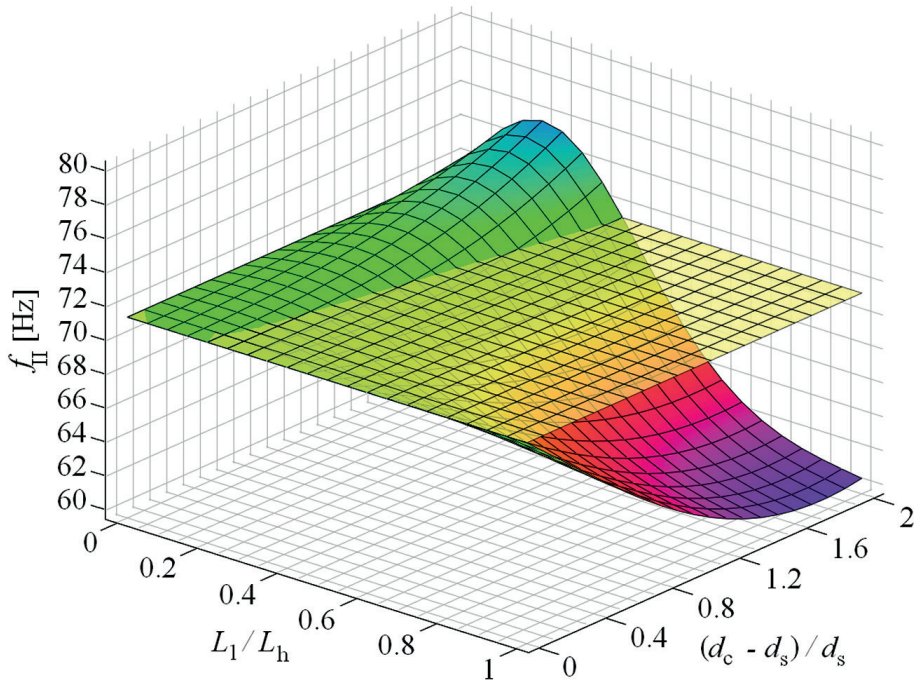

Fig. 5. Dependence of the second transverse natural frequency on the relative height of local coatings and on the relative thickness of local coatings, at the determined material parameters of these coatings

the paper. Values of the first (Fig. 4) and the second (Fig. 5) frequency of the spring without coatings (horizontal yellow plane) are also marked in these diagrams. As can be seen, coatings can increase or decrease the natural frequencies of transverse vibrations depending on their geometrical parameters.

Both in the first and second form of transverse vibrations, regardless of the relative thickness of coatings (within the analyzed example range), such a relative height of coatings can be selected for which the frequencies of these vibrations will not change in relation to the analogous frequencies of the spring without coatings. Thus, the model developed allows for the introduction of essential modifications to the natural frequen- cies of transverse vibrations by means of coatings. In the analyzed example, the difference between the maximum $f_{\max }$ and $\operatorname{minimum} f_{\min }$ frequency value, related to the frequency of the spring without coatings $f_{w c}$, was above $26 \%$ in the case of the first frequency (Fig.3), and exceeded $21 \%$ in the case of the second frequency (Fig.4).

It is obvious that the application of coatings also influences the spring static compression stiffness, defined as a quotient of the static axial force and the corresponding absolute deflection of the spring. Using this definition, after its elementary rearrangements, the dependence between the static compression stiffness $c_{w c}$ of the spring with local coatings and the static compression stiffness $c_{n c}$ of the analogous spring without coatings can be written as follows:

$$
\frac{c_{w c}}{c_{n c}}=\frac{L_{h}}{L_{h}+L_{1}\left(\frac{\gamma_{02}}{\gamma_{01}}-1\right)} .
$$

The diagram of the stiffness quotient described by (30) is presented in Fig. 6 as a function of the relative height and the relative thickness of local coatings, at the given material parameters of these coatings. It can be observed, when analyzing the diagrams in Fig. 4 and Fig. 5, that in the cases where damping coatings cover the spring wire on its whole length, independently of their relative thickness $\left(d_{c}-d_{s}\right) / d_{s}$ (within the analyzed range), they cause a decrease in the natural transverse frequencies, while short coatings always cause a frequency increase when compared to the natural frequencies of the spring without coatings.

When comparing the diagrams in Fig. 4, Fig. 5 and Fig. 6, it is visible that an increase in natural frequencies is not necessarily related to a significant increase in the spring static stiffness.

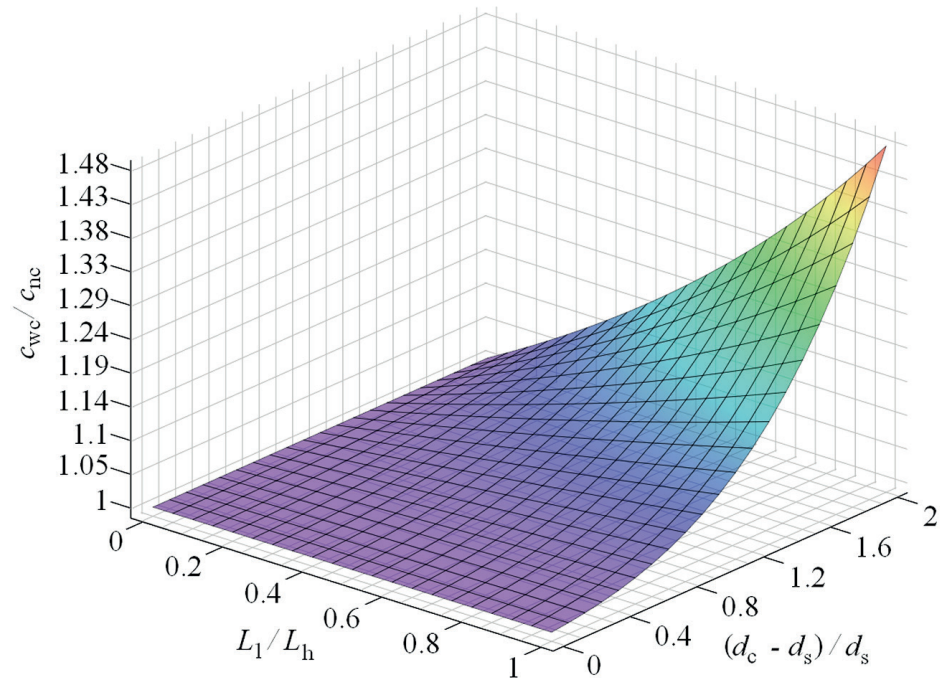

Fig. 6. Stiffness quotient $c_{w c} / c_{n c}$ as dependent on the relative height and relative thickness of local coatings at the given material parameters of these coatings 


\section{Conclusions}

Coatings covering the spring wire along its whole length or in sections can be applied in order to protect the wire against unfavorable factors such as corrosion or abrasive wear as well as in order to increase damping in the system. Thus, it is important to determine the influence of such coatings on spring static and dynamic properties such as stiffness and natural frequencies as well as modes of spring transverse vibrations. In the existing literature there is no study dealing with this problem. The novel calculation model allowing users to determine the frequencies and modes of natural transverse vibrations of helical springs with elastic coatings, subjected to the static axial force, is presented in the paper. It has been shown that coatings of materials with parameters similar to the typical high-molecular plastics applied in machine building can significantly influence the values of natural frequencies. Comparing the results of the model presented herein with the FEM analysis indicates its high accuracy. Attention was drawn to the fact that for the same resultant spring stiffness it is possible to obtain either a decrease or an increase in the frequency of natural transverse vibrations. Thus, the model presented herein enables the selection of material and geometrical parameters of coatings, ensuring that the required properties of the spring being designed are obtained. This can be also useful when coating springs that already exist.

\section{REFERENCES}

[1] S.V. Sorokin, "Linear dynamics of elastic helical springs: asymptotic analysis of wave propagation", Proc. R. Soc. A 465, 1513-1537 (2009).

[2] S. Wang, S. Lei, J. Zhou, and H. Xiao, "Mathematical model for determination of strand twist angle and diameter in stranded-wire helical springs", Journal of Mechanical Science and Technology 24 (6), 1203-1210 (2010).

[3] J. Snamina and B. Sapiński, "Energy balance in self-powered MR damper-based vibration reduction system", Bull. Pol. Ac.: Tech. 59 (1), 75-80 (2011).

[4] D.S. Yantek and M.J. Lowe, "Analysis of a mechanism suspension to reduce noise from horizontal vibrating screens", Noise Control Engineering Journal 59 (6), 568-580 (2011).

[5] W.T Mayers, "Helical coil spring damper assemblies", US Patent, 4538563A, 1985.

[6] S. Nix, "Schubfeder", DE Patent, 10104936A1, 2002.

[7] C. Berger and B. Kaiser, "Results of very high cycle fatigue tests on helical compression springs", International Journal of Fatigue 28, 1658-1663 (2006).

[8] J. Krużelecki and D. Szubartowski, "Influence of support compliances on stability and limiting slenderness ratio of compression helical springs", Journal of Theoretical and Applied Mechanics 52 (1), 81-92 (2014).

[9] C. Muzio, "Coated coil spring, particularly for automotive suspensions", Patent, EP0878637A3, 1999.

[10] K. Michalczyk, "Influence of the elastomeric coating on parameters of steady state vibrations of coil springs in the resonance and outside it", Journal of Theoretical and Applied Mechanics 52 (2), 507-518 (2014).
[11] K. Michalczyk, "Dynamic stresses in helical springs locally coated with highly-damping material in resonant longitudinal vibration conditions", International Journal of Mechanical Sciences 90, 53-60 (2015).

[12] A.E.M. Love, "The propagation of waves of elastic displacement along a helical wire", Transactions of the Cambridge Philosophical Society 18, 364-374 (1899).

[13] W.H. Wittrick, "On elastic wave propagation in helical springs", International Journal of Mechanical Sciences 8, 25-47 (1966).

[14] J.E. Mottershead, "Finite elements for dynamical analysis of helical rods", International Journal of Mechanical Sciences 22, 267-283 (1980).

[15] B. Tabarrok, A.N. Sinclair, M. Farshad, and H. Yi, "On the dynamics of spatially curved and twisted rods - a finite element formulation", Journal of Sound and Vibration 123(2), 315-326 (1988).

[16] N. Stander and R.J. Du Preez, "Vibration analysis of coil Springs by means of isoparametric curved beam finite elements", Communications in Applied Numerical Methods 8, 373-383 (1992).

[17] M. Taktak, F. Dammak, S. Abid, and M. Haddar, "A finite element for dynamic analysis of a cylindrical isotropic helical spring", Journal of Materials and Structures 3, 641-658 (2008).

[18] D. Pearson, "The transfer matrix method for the vibration of compressed helical springs", Journal of Mechanical Engineering Science 24, 163-171 (1982).

[19] V. Yildrim, "Investigation of parameters affecting free vibration frequency of helical springs", International Journal for Numerical Methods in Engineering 39, 99-114 (1996).

[20] L.E. Becker, G.G. Chassie, and W.L. Cleghorn, "On the natural frequencies of helical compression springs", International Journal of Mechanical Sciences 44, 825-841 (2002).

[21] J.A. Haringx, "On highly compressible springs and rubber rods, and their application for vibration-free mountings", II. Philips Research Reports 4, 49-80 (1949).

[22] J. Krużelecki and M. Życzkowski, "On the concept of an equivalent column in the problem of stability of compressed helical springs", Ingenieur-Archiv 60, 367-377 (1990).

[23] V. Yildrim, "An efficient numerical method for predicting the natural frequencies of cylindrical helical springs", International Journal of Mechanical Sciences 41, 919-939 (1999).

[24] W.H. Wittrick and F.W. Williams, "A general algorithm for computing natural frequencies of elastic structures", Quarterly Journal of Mechanics and Applied Mathematics 24, 263-284 (1971).

[25] D. Pearson and W.H. Wittrick, "An exact solution for the vibration of helical springs using a Bernoulli-Euler model", International Journal of Mechanical Sciences 28, 83-96 (1986).

[26] J. Lee and D.J. Thompson, "Dynamic stiffness formulation, free vibration and wave motion of helical springs", Journal of Sound and Vibration, 239 297-320 (2001).

[27] J. Lee, "Free vibration analysis of cylindrical helical springs by the pseudospectral method", Journal of Sound and Vibration 302, 185-196 (2007).

[28] A.M. Yu and Y. Hao, "Improved Riccati transfer matrix method for free vibration of non-cylindrical helical springs including warping", Shock and Vibration 19, 1167-1180 (2012).

[29] S. Timoshenko, Theory of elastic stability, McGraw Hill, New York and London, 1936.

[30] J.A. Haringx, "On highly compressible springs and rubber rods, and their application for vibration-free mountings I." Philips Research Reports 4, 401-449 (1948).

[31] A.R. Guido, L. Della Pietra, and S. Della Valle, "Transverse vibrations of helical springs”, Meccanica 13 (2), 90-108 (1978). 
[32] L. Della Pietra and S. Della Valle, "On the dynamic behavior of axially excited helical springs", Meccanica 17, 31-43 (1982).

[33] Ch. Flenker and U. Uphoff, "Efficient Valve-Spring Modelling with MBS Valve-Train Design"' MTZ 66, 946-950 (2005).

[34] V. Kobelev, "Effect of static axial compression on the natural frequencies of helical springs", Multidiscipline Modeling in Materials and Structures 10, 379-398 (2014).

[35] K. Michalczyk, "Analysis of lateral vibrations of the axially loaded helical spring", Journal of Theoretical and Applied Mechanics 53 (3), 754-755 (2015).

[36] N.G. Stephen and S. Puchegger, "On the valid frequency range of Timoshenko beam theory", Journal of Sound and Vibration 297, 1082-1087 (2006).

[37] L. Majkut, "Free and forced vibrations of Timoshenko beams described by single difference equation", Journal of Theoretical and Applied Mechanics 47 (1), 193-210 (2009).
[38] I.C. Finegan and R.F. Gibson, "Recent research on enhancement of damping in polymer composites", Composite Structures 44, 89-98 (1999).

[39] B. Benchekchou, M. Coni, H.V.C. Howarth, and R.G. White, "Some aspects of vibration damping improvement in composite materials", Composites Part B: Engineering 29 (6), 809-817 (1998).

[40] D.D.L Chung, "Structural composite materials tailored for damping", Journal of Alloys and Compounds 355, 216-223 (2003).

[41] A. Kęsy and J. Kotliński, "Mechanical properties of parts produced by using polymer jetting technology", Archives of Civil and Mechanical Engineering X (3), 37-50 (2010).

[42] D.W. Van Krevelen and K. te Nijenhuis, Properties of Polymers: Their Correlation with Chemical Structure; their Numerical Estimation and Prediction from Additive Group Contributions, Elsevier, Amsterdam, 2009.

[43] C.M. Harris and A.G. Piersol, Harris'shock and vibration handbook, McGRAW-HILL, New York, 2002. 